\title{
Olivier Crasset, La Santé des artisans
}

Rennes, Presses universitaires de Rennes, 2017

\section{Julien Gros}

\section{(2) OpenEdition}

\section{Journals}

Édition électronique

URL : http://journals.openedition.org/travailemploi/7736

DOI : 10.4000/travailemploi.7736

ISSN : 1775-416X

Éditeur

DARES - Ministère du Travail

Édition imprimée

Date de publication : 1 juillet 2017

Pagination : 110-113

ISSN : 0224-4365

Référence électronique

Julien Gros, «Olivier Crasset, La Santé des artisans », Travail et Emploi [En ligne], 151 | juillet-septembre 2017, mis en ligne le 11 juillet 2019, consulté le 25 septembre 2020. URL : http://

journals.openedition.org/travailemploi/7736 ; DOI : https://doi.org/10.4000/travailemploi.7736 


\section{La santé des artisans}

\section{Olivier Crasset}

Rennes, Presses universitaires de Rennes, 2017, 290 p.

\section{Lu par Julien Gros*}

Ce livre propose une stimulante mise en perspective sociologique du travail des artisans, qui résonne particulièrement avec l'actualité du monde du travail. En effet, le travail indépendant a récemment fait en France l'objet de réformes profondes, qui se sont accompagnées d'un net regain d'intérêt des sociologues à son égard ${ }^{1}$. Dans ce cadre, l'ouvrage d'Olivier Crasset est consacré à un groupe que l'éclat des formes renouvelées de travail indépendant - auto-entrepreneuriat, travailleurs indépendants des plateformes numériques - pourrait dissimuler, un de ces groupes professionnels auxquels on réserve souvent le vocable ambigu de « traditionnel » et qui continue de représenter une part essentielle des indépendants, auto-entrepreneurs ou non.

L'auteur, O. Crasset, est docteur en sociologie de l'université de Nantes ${ }^{2}$, haut lieu, depuis longtemps, de l'étude empirique des mondes ouvriers. Et l'auteur s'inscrit dans cette filiation par l'enquête remarquable qu'il consacre aux artisans, cette fraction des indépendants qui relève du « travail des choses ${ }^{3}$ »-j'accorde les termes au masculin dans la mesure où, j'y reviendrai, c'est en fait d'hommes dont il est question. Cette enquête tire parti de l'expérience antérieure d'O. Crasset, qui a lui-même été ferronnier d'art : une très bonne intégration dans le milieu de l'artisanat breton et une connaissance par corps du métier en constituent donc la sève. Cependant, le matériau n'est absolument pas un récit d'expérience, puisque l'auteur adopte un protocole empirique particulièrement riche, constitué d'entretiens ethnographiques et d'observations participantes, auxquels s'ajoutent quelques traitements statistiques réalisés à partir de l'enquête Santé et itinéraire professionnel. L'enquête de terrain fait l'objet d'une analyse réflexive exposée dans l'introduction, qui permet à O. Crasset d'utiliser avec

\footnotetext{
* Université Paris Dauphine, Institut de recherche interdisciplinaire en sciences sociales (Irisso).

1. Abdelnour S. (2017), Moi, petite entreprise. Les auto-entrepreneurs, de l'utopie à la réalité, PUF, coll. « Humensis »; Bernard S., Dressen M. (2014), « Penser la porosité des statuts d'emploi », La Nouvelle Revue du travail, $\mathrm{n}^{\circ}$ 5, mis en ligne le 11 décembre 2015, consulté le 7 décembre 2017. URL : http://nrt.revues.org/1830 ; AMOSSÉ T. (2012), « Diriger une PME. Introduction », Travail et Emploi, n 130, pp. 5-8 ; ABDELNOuR S., BERNARD S., GROS J., « Genre et travail indépendant. Place des femmes et divisions sexuées dans le non-salariat », Travail et Emploi, $\mathrm{n}^{\circ} 150$.

2. Cet ouvrage est tiré de sa thèse, soutenue en 2014.

3. Verret M. (1995), Chevilles ouvrières, Paris, Les Éditions de l'Atelier.
} 
rigueur les lunettes que lui fournit son expérience. La conséquence directe de cette proximité de l'objet est une empathie forte et féconde envers ses enquêtés.

Cet ouvrage s'inscrit dans la continuité d'études sur l'artisanat, après celle de Bernard Zarca, menée dans les années 1970 et consacrée à la constitution de l'artisanat en groupe social, ou celle de Caroline Mazaud sur la diffusion de la figure du chef d'entreprise gestionnaire ${ }^{4}$. L'auteur choisit d'approcher l'artisanat par la santé de ses travailleurs et s'intéresse plus précisément à l'interaction entre parcours professionnels et santé. Il se donne pour but - même si le livre le dépasse en fait très largement - de résoudre le paradoxe de l'apparente bonne santé des artisans, qui connaissent pourtant, au sein d'un même domaine d'activité, des conditions de travail souvent aussi dures que celles des ouvriers.

Le premier chapitre définit les contours de l'analyse. L'artisanat est l'objet de multiples définitions concurrentes, émanant de groupes (syndicats, administration fiscale, statisticiens) aux intérêts variés. L'auteur ne prétend pas fournir la bonne définition de l'artisanat, mais il en propose une, qu'il admet fort justement dépendre des questions qu'il souhaite explorer : les artisans sur lesquels porte son analyse sont des travailleurs indépendants dont le métier implique un travail manuel et qui prennent directement part à la production (qu'ils emploient ou non des salariés, même si ceux-ci sont toujours, par définition, en nombre limité). C'est à leur propos qu'il forge la notion de capital corporel, un apport conceptuel intéressant du livre.

Son développement se déploie en deux grands moments. Tout d'abord, un long chapitre replace les conditions de travail de l'artisan dans les différents collectifs auxquels ce dernier prend part. Ensuite, quatre chapitres retracent, étape par étape, les relations entre travail et santé aux différents stades du parcours (ou de la carrière) de l'artisan. C'est dans ces chapitres, plus attendus mais non moins intéressants, qu'est le plus maniée et exploitée la notion de capital corporel, ce capital que l'artisan constitue, dépense puis convertit en d'autres capitaux lui permettant de limiter l'effet du travail sur le corps (capital économique et capital social), cela dans des conditions variables et inégalitaires. Par cette notion, l'auteur met en évidence le fait que le rapport au corps et à son usure est socialement situé et qu'il dépend d'autres espèces de capitaux. Cela le conduit à différencier, au sein de sa population, les artisans d'extraction populaire et les artisans-créateurs issus de catégories mieux dotées culturellement (qui se rapprochent des artisans d'art étudiés par Anne Jourdain et des agriculteurs néo-ruraux étudiés par Madlyne Samak ${ }^{5}$.

Mais je laisse de côté cette notion (qui est clairement l'apport le plus souligné du livre et sur lequel Olivier Schwartz insiste dans la préface) pour insister sur deux autres points abordés par l'auteur et qui me semblent engager des pistes stimulantes et importantes.

4. ZARCA B. (1986), L'Artisanat français, du métier traditionnel au groupe social, Economica ; MAZAUD C. (2013),

L'Artisanat français. Entre métier et entreprise, Presses universitaires de Rennes, coll. « Le sens social ».

5. Voir, par exemple, Jourdain A. (2017), « Des artisans d'art aux artisanes d'art » et SAMAK M., « Le prix du "retour" chez les agriculteurs "néo-ruraux" », Travail et Emploi, $\mathrm{n}^{\circ} 150$. 
Le premier concerne les développements sur la dimension collective du travail des artisans présentés dans le cinquième chapitre. L'auteur y documente avec précision plusieurs interactions dans lesquelles les artisans sont pris au cours de leur travail - sur les chantiers qu'ils dirigent ou sur lesquels ils interviennent comme sous-traitants, mais aussi dans le fonctionnement interne de l'entreprise. Un des résultats majeurs de ce chapitre est le caractère ambivalent de l'embauche de salariés. En effet, contrairement à ce qu'on pourrait attendre, embaucher des salariés n'a pas mécaniquement pour effet de libérer l'employeur des tâches les plus difficiles. L'auteur observe même souvent le contraire. La présence de salariés sur les chantiers expose l'employeur à davantage de pénibilité, puisqu'il ne peut confier au salarié les activités qui leur sont interdites car trop pénibles ou dangereuses. "Il vaut mieux que ce soit moi qui tombe qu'un salarié », lui dit un enquêté (p. 139) : la protection dont jouit le salarié trouve son revers dans la liberté juridiquement plus grande qu'a le travailleur indépendant de se mettre en danger. En outre, continuer à réaliser les tâches les plus pénibles permet aux artisans employeurs de conserver une forme de supériorité hiérarchique en prouvant leur maîtrise technique du métier. Enfin, pour nombre d'entre eux, l'emploi de salariés engendre un accroissement de la pénibilité psychologique : un stress et une fatigue dont certains se libèrent quand ils en ont les moyens, à l'approche de la retraite. Cela dit, l'embauche peut aussi donner à l'artisan l'opportunité de déplacer son activité vers la gestion et de consacrer plus de temps à des tâches gratifiantes et moins usantes physiquement : visites au domicile des futurs clients ou chez les fournisseurs de matériel, rôle d'expert et de conseil, repas au restaurant avec les confrères. Finalement, l'espace dont rend compte O. Crasset est une zone de frottement, méconnue, dans laquelle l'embauche de salariés, même si elle permet à l'artisan de goûter à une forme de promotion sociale, ne le libère pas de la pénibilité et des traits ouvriers de son activité.

L'autre dimension qui me semble constituer une piste intéressante est l'analyse que l'auteur propose de la place des femmes dans ce monde masculin. Il prend soin de bien distinguer ces femmes selon qu'elles sont des cheffes d'entreprises ou des conjointes de chef d'entreprises, en les désignant par deux notions différentes, ce qui n'est pas souvent le cas dans la littérature sur les « indépendantes » ${ }^{7}$. En outre, il propose une description particulièrement intéressante du rôle de la femme du patron, la figure de la « patronne » qui, quoique dominée (elle n'est patronne que parce qu'elle est la femme du patron), occupe une position d'autorité. C'est particulièrement le cas sur ce qui touche à la santé, où son autorité s'étend sur les salariés, mais également sur son conjoint. L'auteur documente ici un versant du « paternalisme » peu connu, où les dispositions construites comme féminines jouent dans l'encadrement du collectif de

\footnotetext{
6. Sa volonté de se limiter aux artisans qui prennent encore part à la production, si elle lui fait mettre le doigt sur cette situation, lui impose de laisser de côté la question des transformations induites par le passage du travailleur indépendant au patron-gestionnaire. Voir Amossé T. (2012), « Diriger une PME. Introduction », art. cit., et MAZAUD C. (2013), L'Artisanat français, du métier traditionnel au groupe social, op. cit.

7. Voir Abdelnour S., Bernard S., Gros J. (2017), « Genre et travail indépendant. Place des femmes et divisions sexuées dans le non-salariat », art. cit.
} 
travail, que la patronne materne : « Les salariés, c'est comme les gamins, il faut serrer les boulons », lui dit une enquêtée (p. 149). En revanche, si les femmes du monde de l'artisanat œuvrent à la bonne santé de ces hommes lorsqu'elles sont conjointes, ce n'est absolument pas le cas lorsqu'elles sont elles-mêmes cheffes d'entreprise. Elles sont alors autant que les hommes concernées par la mise à l'épreuve du corps et par la mise en scène d'une virilité - parfois redoublée, précisément, pour tenir leur place dans ce milieu d'hommes.

Ce développement, parce qu'il est très riche, laisse en certains endroits un goût d'inachevé. En effet, l'analyse semble à même d'ouvrir un questionnement plus vaste sur les rôles croisés du statut d'emploi et du genre dans la division du travail. En particulier, l'analyse nous invite, sans que l'auteur le dise, à nous demander comment l'artisanat a été construit comme un monde aussi masculin et, ce qui est sans doute lié, comme un monde où le capital corporel est si central. Pour ne prendre qu'un exemple, dès la définition du périmètre de son étude, l'auteur juge nécessaire pour être un artisan le fait de travailler « manuellement », «physiquement », « musculairement » (les trois mots sont utilisés par l'auteur). Bien sûr, cette centralité du travail manuel est constitutive de l'artisanat et l'auteur devait la reprendre : le chef de l'entreprise artisanale est l'homme de métier qui travaille de ses mains, et bien peu souvent la gestionnaire et comptable qu'est sa conjointe. Mais elle est également très genrée et située socialement : l' «énergie musculaire » fournie n'a pas le même sens selon les métiers ; elle peut y être forte mais inégalement valorisée, et ainsi entrer inégalement dans la définition du métier. Le fait de définir l'artisanat en rendant centrale cette caractéristique un peu floue qu'est le travail manuel a-t-il à voir avec sa construction comme un monde masculin ? L'auteur aurait pu soulever la question sans même chercher à la résoudre. Cela aurait sans doute permis d'expliciter les raisons, légitimes, qui font que le livre porte sur les hommes artisans, que le capital corporel dont il est question est fortement sexué et que le masculin du titre n'est pas un masculin neutre. Mais il faut immédiatement préciser que ces difficultés concernent la quasi-totalité des chercheur.e.s qui écrivent sur les travailleur-ses indépendant·e.s, y compris l'auteur de ce compte-rendu. Et c'est un grand mérite d'O. Crasset que de nous inviter à nous poser la question de manière plus précise. Quitte à nous donner les outils pour finalement constater qu'elle reste entière chez lui.

Au bout du compte, ce livre apportera beaucoup à celles et ceux qui s'intéressent aux évolutions contemporaines du travail indépendant ou à la santé au travail. Parmi ces personnes, celles qui travaillent à partir d'enquêtes statistiques trouveront également des éléments intéressants. Ainsi, l'auteur aide à comprendre comment l'« effet travailleur sain » échappe plus à l'enquête par questionnaire quand il s'agit d'indépendant·e·s que de salarié.e.s : le salariat a institutionnalisé un lien entre travail

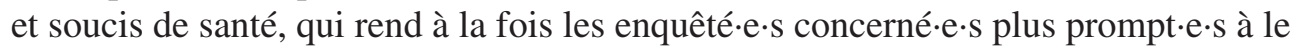
déclarer et les questionnaires plus aptes à le saisir. Il donne enfin des éléments pour mieux réfléchir à ce qui se joue, en termes de mobilité sociale, dans ces fractions du monde du travail que l'absence du salariat rend plus difficile à décrire. 